\title{
Nonlinear Lifting Line Theory Applied To Vertical Axis Wind Turbines: Development of a Practical Design Tool
}

\author{
David Marten $^{1 *}$, George Pechlivanoglou ${ }^{1}$, Christian Nayeri ${ }^{1}$, Christian Oliver Paschereit ${ }^{1}$
}

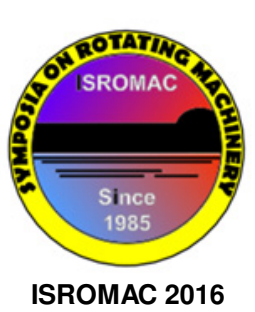

International

Symposium on Transport

Phenomena and

Dynamics of

Rotating Machinery

Hawaii, Honolulu

April 10-15, 2016

\begin{abstract}
Recently a new interest in vertical axis wind turbine (VAWT) technology is fueled by research on floating support structures for large scale offshore wind energy application. For the application on floating structures at multi megawatt size, the VAWT concept may offer distinct advantages over the conventional horizontal axis wind turbine (HAWT) design. As an example VAWT turbines are better suited for upscaling and, at multi megawatt size, the problem of periodic fatigue cycles reduces significantly due to a very low rotational speed. Additionally, the possibility to store the transmission and electricity generation system at the bottom, compared to the tower top as in a HAWT, can lead to a considerable reduction of material logistics costs. However, as most VAWT research stalled in the mid 90's [1], no established and sophisticated tools to investigate this concept further exist today. Due to the complex interaction between unsteady aerodynamics and movement of the floating structure fully coupled simulation tools, modelling both aero- and structural dynamics are needed. A nonlinear Lifting Line Free Vortex Wake code was recently integrated into the open source wind turbine simulation suite QBlade. This paper describes some of the necessary adaptions of the algorithm, which differentiates it from the usual application in HAWT simulations. A focus is set on achieving a high robustness and computational efficiency. A short validation study compares simulation results with those of a U-RANS and a Double Multiple Streamtube (DMS) simulation.
\end{abstract}

\footnotetext{
Keywords

Wind Turbine Aerodynamics — Numerical Simulation — VAWT

${ }^{1}$ Chair of Fluid Dynamics, Hermann Föttinger Institute, TU Berlin, Berlin, Germany

*david.marten@tu-berlin.de
}

\section{INTRODUCTION}

The aerodynamics and the flow field around a VAWT are very complex. During one revolution of the rotor the angle of attack, and thus the blade loads, are constantly changing. Additionally, at low tip speed ratios (TSR), the blades experience dynamic stall at certain azimuthal positions. This inherent unsteadiness during operation is the main reason why no practical, efficient and robust design tool exists for VAWT, which is comparable to the very popular blade element momentum (BEM) method, widely used for HAWT design and certification.

Paraschivoiu [2] developed the double multiple stream tube algorithm (DMS), as an extension of the BEM method, for VAWT. In contrast to the BEM the DMS algorithm employs two actuator discs, one for the upstream and one for the downstream half circle of rotation. This method is used to predict the performance of VAWT in the early design stage, however for high blade loading, due to high solidities or a high TSR [3] convergence problems arise. One problem is that momentum balance based codes assume a steady state flow field and this assumptions is even more violated for VAWT than for HAWT rotors. On the other hand Reynoldsaveraged Navier-Stokes (RANS) or Large Eddy (LES) CFD simulations are still too computationally expensive to be used as a design tool in coupled dynamics simulations. Being of intermediate complexity and physical accuracy Lifting Line Theory (LLT) based codes, combined with a free vortex wake model, are a promising candidate for a capable
VAWT design tool. The Lifting Line Theory captures the unsteady blade loading, whereas the complex wake structure (see Figure 1) is modeled through free evolving vortex elements. LLT based codes have been successfully applied to VAWT simulation (as by Ferreira [4]), but up to date no such code, dedicated to VAWT, is commercially or publicly available.

This paper describes a newly developed, efficient and robust LLT based design and simulation tool for VAWT. An unsteady LLT simulation module for VAWT is integrated with the open source wind turbine simulation tool QBlade [5]. As a result of this integration, advanced simulations can be setup using the airfoil, polar, turbulent wind field and blade database of QBlade. Additional aerodynamic models for the tower deficit and ground effects have been implemented (details in [6]). Furthermore the turbine model is capable of prescribed 6 degree of freedom movement enabling an easy coupling to other dynamics simulation modules. The focus of this paper is on the specific differences and treatments that distinguish the implementation of a LLT code for VAWT from an implementation for HAWT rotors and how these affect its robustness, efficiency and accuracy. Moreover the performance of the code will be demonstrated in a validation study, comparing the obtained results to published data from a CFD and a DMS simulation. The resulting software is freely distributed under an open source license. The code can be found under: sourceforge.net/projects/qblade. 


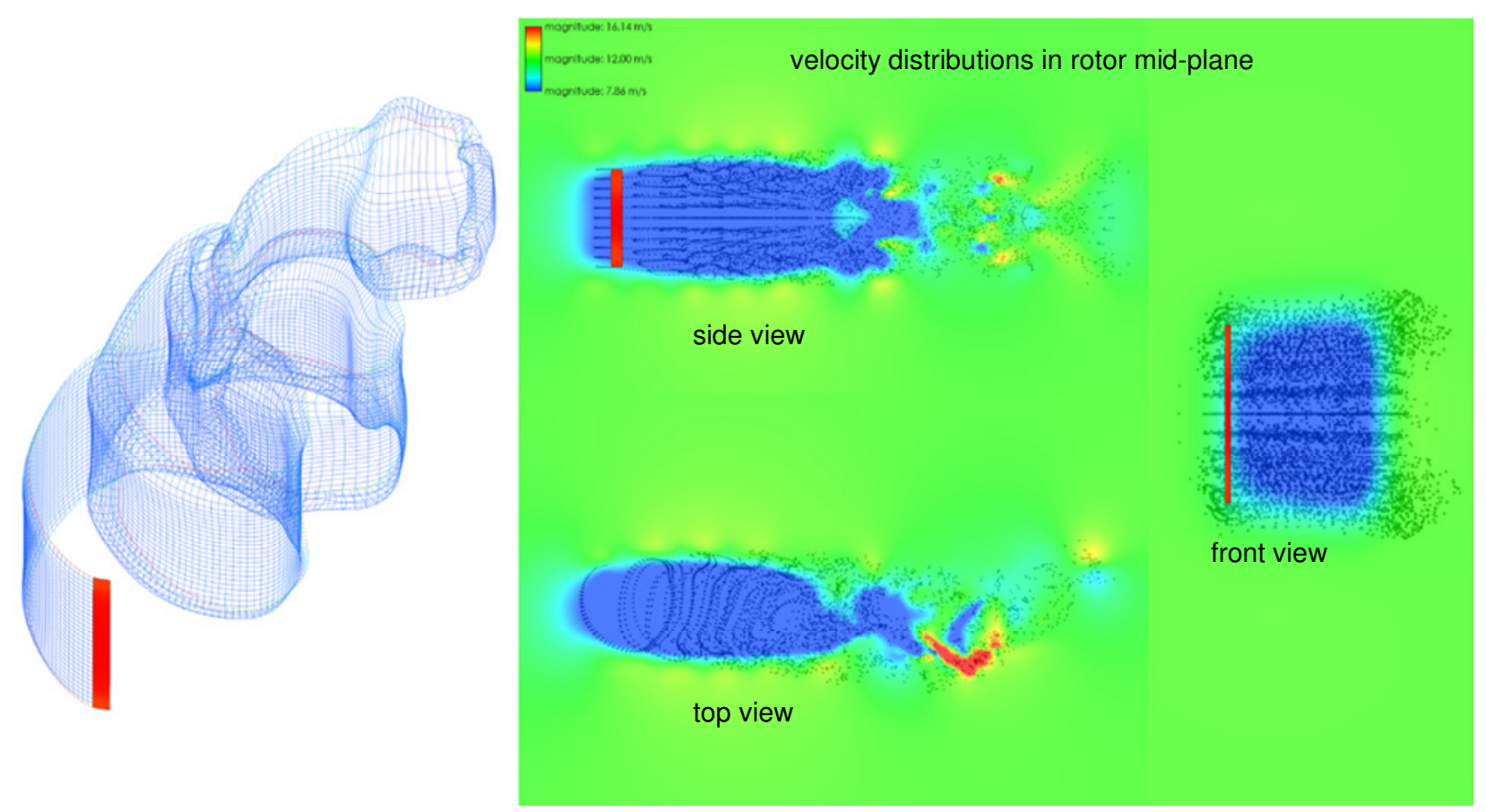

Figure 1. Left: Detail of free evolving VAWT wake, right: velocity distributions for high induction / high tip speed ratio case

\section{LLT MODIFICATIONS FOR VAWT SIMULATIONS}

Recently a nonlinear lifting line free vortex wake algorithm has been implemented in QBlade. The LLT is a very general simulation method that can be used to model both HAWT and VAWT rotors with an almost identical formulation. Blade forces are calculated using the lifting line formulation where the circulation is modeled through distributed vortices, located at the quarter chord positions of the blade (see Figure 2). The circulation is calculated from tabulated airfoil data and the blade inflow angles that are computed under the influence of the free wake induction. The wake is described by freely floating vortex elements that are shed from the trailing edge during every time step.

Compared to BEM based methods which model the wake with a momentum balance across the rotor disc this is a much more physically sound approach that relies on far less assumptions.

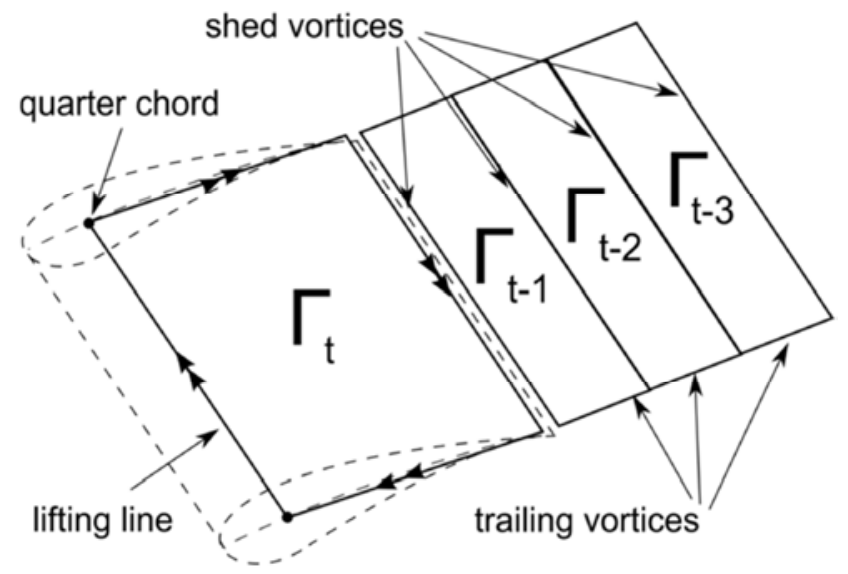

Figure 2. Geometry of a blade panel, position of lifting line, shed and trailing vortex elements
As the history of the flow is always contained in the wake, in the form of previously shed wake elements, this method is especially suited to model transient turbine behavior, such as start- or ramp up cases or any situation involving relative motion of the whole rotor. Especially in these transient cases a significant improvement, compared to BEM based models, can be expected. An additional advantage is that, due to the explicit wake modeling, the simulation results also contain the velocity distribution around the rotor, allowing to extract wake deficit profiles or induced velocity distributions around the rotor (Figure 1). Because the LLT is also based on far less assumptions than momentum balance based methods, generally a far lower number of empirical corrections are required. As an example: the effect of blade tip loss is already included in the LLT formulation whereas momentum balance based codes rely on a tip loss correction. Furthermore, the convergence problems that momentum balance based methods are often facing are not an issue with LLT based codes as they can even model situations of flow reversal within the rotor. This robustness, combined with a reasonable computational cost (compared to CFD methods), makes the LLT a very promising candidate as a universal design method for VAWT.

More general details about the implemented LLT method can be found in the report of van Garrel [7], details about the implementation in QBlade have been published in [5]. The aim of this paper is to give some details about the specifics that differentiate the implementation for VAWT simulation from that for HAWT simulation.

\subsection{Calculation of relative blade velocities and azimuthal discretization}

During every time step an angle of attack $(A \circ A)$ needs to be calculated at every blade section. As this AoA determines the lift and drag coefficients, stored in data tables, and thus the 
forces acting on the respective blade section, it has a very large effect on the predicted performance of the rotor. The AoA is evaluated at the quarter chord position of each blade panel from the total velocity that the blade experiences. The total blade velocity is a superposition of inflow velocity, induced velocity, due to wake and rotor induction and a relative velocity component due to the blades' movement.

$$
V_{\text {tot }}=V_{\text {ind }}+V_{\text {rel }}+V_{\text {in }}
$$

In most implementations of the LLT for HAWT applications the velocity component due to blade movement is computed via a simple derivative of the blades' position in time $[7,8]$. This practice is usually accurate enough for HAWT, where a $10^{\circ}$ azimuthal discretization is usually recommended [9]. The error between the actual tangential velocity and the approximation through the derivation does not have a very large effect on evaluated $A \circ A$ as the circular path of the blade is usually perpendicular to the inflow. For VAWT however the inflow velocity is orthogonal to the axis of rotation and thus the tangential velocity vector due to blade movement lies in plane with the inflow. In this case the error that is introduced due to the first order derivative has a very large effect on the computed AoA (Figure 3) and using the derivation procedure makes the simulation very sensitive to the chosen azimuthal discretization.

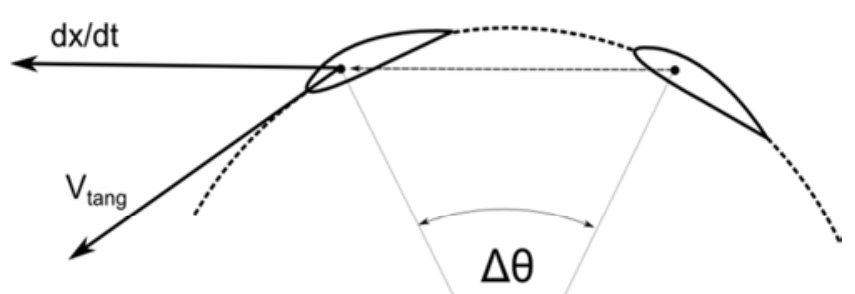

Figure 3. Time derivative of position vector vs tangent vector for a VAWT
To circumvent this problem, the relative velocity is split up into a tangential component (due to the blades rotation) and a component describing all other blade movements (such as structural deformations or floater movements).

$$
V_{\text {rel }}=V_{\text {tang }}+V_{\text {mov }}
$$

The tangential component is constructed via a unit tangent vector and the known angular velocity magnitude. The component describing all other blade movements is then calculated by derivation. From this component the part of the blades' velocity due to rotation of the rotor has to be subtracted, as it is already included in the, now separate, tangential component. This is done by reconstructing the blades' angular position of the last time step for the current rotor axis and using this position in the derivative. This treatment makes the simulation results very insensitive to the chosen azimuthal discretization and reasonable results can be obtained with an azimuthal discretization of up to $20^{\circ}$ (see Figure 4). The advantage of this velocity decomposition, when compared to higher order time discretization, is the significantly lower computational cost.

\subsection{Wake treatment}

The computational cost of the LLT method is situated between the cost of momentum balances based methods and those of CFD. To be used in certification test like scenarios, where several million aerodynamic time steps are required, a high efficiency is of utmost importance. The driving factor for the computational cost of the LLT method is the number of free vortex elements, contained in the wake. To simulate the free evolution of the wake, each wake element has to be convected under the influence of induction from all other wake elements thus the computational time needed to run a simulation scales roughly linearly with the squared number of free vortex elements. As new wake elements are created at the blades trailing edge during every time step the computational cost of the LLT grows exponentially over time when no measures are taken.

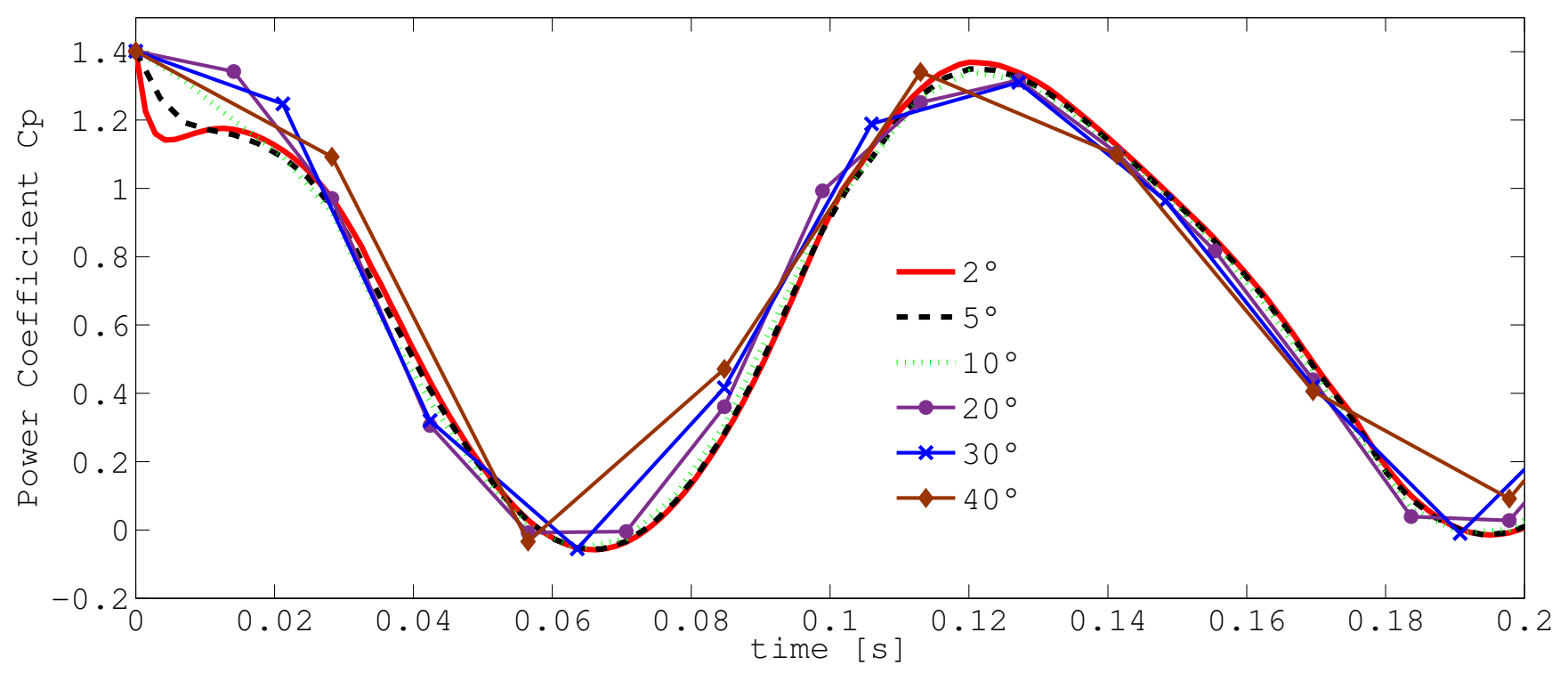

Figure 4. Power coefficient over one rotation for six different azimuthal discretizations 
To enable the implementation of several strategies that reduce the number of wake elements an object orient attachment-detachment scheme has been implemented. This is an important feature of the code as it allows to remove wake elements from the simulation domain, based on arbitrary conditions, without breaking the connectivity of the complex wake mesh.

\subsubsection{Wake truncation}

One practical approach to limit the number of free wake elements to a fixed size is to simply remove all wake elements from the domain after they have reached a certain age (example in Figure 5). For this treatment the vortex age is made dimensionless with the time the rotor needs for a full revolution, thus the wake age, as it is used here, represents the age of a vortex in full revolutions of the rotor.

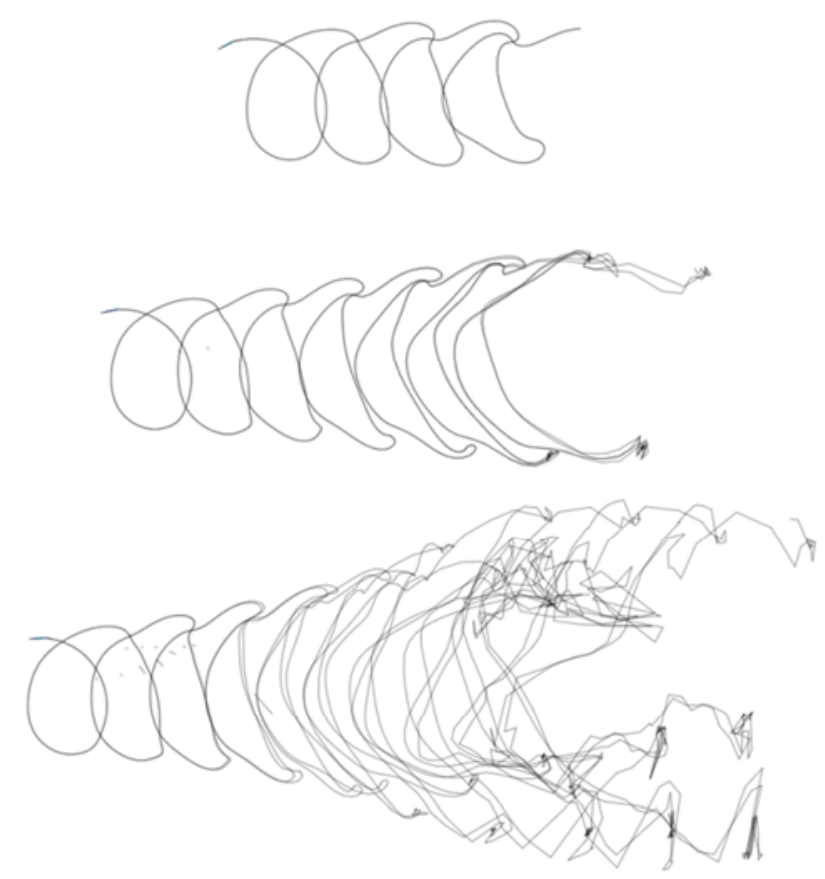

Figure 5. Top view of single blade wake at a TSR of 4 with three different values for wake truncation. From top to bottom: 4,7 , and 10

To demonstrate the effect of this wake truncation on the resulting accuracy of the simulation, several test cases for exemplary tip speed ratios and a range of the parameter governing the maximum vortex age have been conducted. Figure 6 shows the relative error of the resulting averaged power coefficient compared to a baseline simulation, over different values of the wake age. The three values for tip speed ratio that have been chosen represent low (TSR: 2), optimal (TSR: 3.2) and high (TSR: 4) induction cases. The comparison shows that a wake length of around 8 rotor revolutions is an acceptable value, even for higher tip speed ratios, as the relative error due to the wake truncation drops below $1 \%$ for all simulated cases.

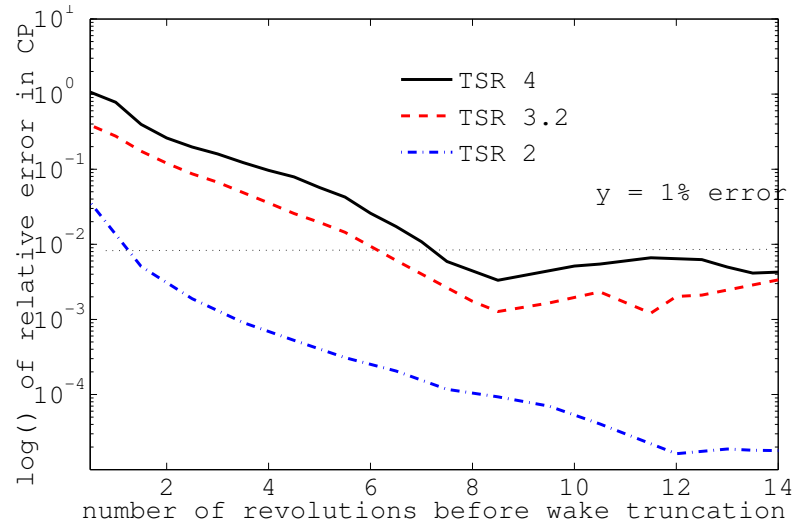

Figure 6. The effect of wake truncation on the simulation accuracy

\subsubsection{Wake reduction}

When free vortex wake methods are applied to simulate HAWT it is common practice to reduce the amount of free vortices by lumping the trailing vortices into concentrated tip vortices and removing the shed vorticity from the wake. This usually takes place at some point sufficiently far away downstream from the rotor disc (as described in [10]). As the trailing vorticity usually is dominant in the wake of a HAWT, the removal of the shed vorticity only has very little effect on the simulation accuracy. Additionally, because the trailing vorticity is conserved, as it is lumped, this is a very effective means of reducing the number of wake elements drastically while still preserving a detailed wake shape several rotor diameters downstream of the rotor plane.

Due to the ever changing angle of attack of a VAWT, and the resulting change in blade circulation, neither the trailing nor the shed vorticity is dominant in the flow field. Both types of vorticity play an equal part in the description of the flow field and thus the strategy as applied to HAWT cannot be applied to VAWT simulations. The strategy that is applied instead is based on the assumption that in every time step there are vortices being shed from the trailing edge that have a more or less negligible circulation. Usually, for straight blade geometries, this is the trailing vorticity, shed from the inner blade regions, or the shed vorticity being shed when the blade is at an azimuthal position with very light loading. Based on this assumption a percentage of vortices is removed right after it is being shed. The amount of vorticity to be removed is a user input. Based on this input the vortices with the lowest absolute circulation, created at the last time step, are removed until the number of vortices that was specified in the user input has been met. To demonstrate the effect that this wake reduction has on the accuracy of the simulation the relative error of the computed power coefficient (averaged over one rotor revolution) is plotted over the percentage of removed wake elements in Figure 7. It can be seen that, regardless of the simulated tip speed ratio, up to $50 \%$ (effectively reducing simulation time by a factor of 4 ) of the vortices can be removed in this way without introducing an error above $1 \%$. Figure 8 shows an exemplary visualization of a wake where $30 \%$ of the vortices have been removed. 


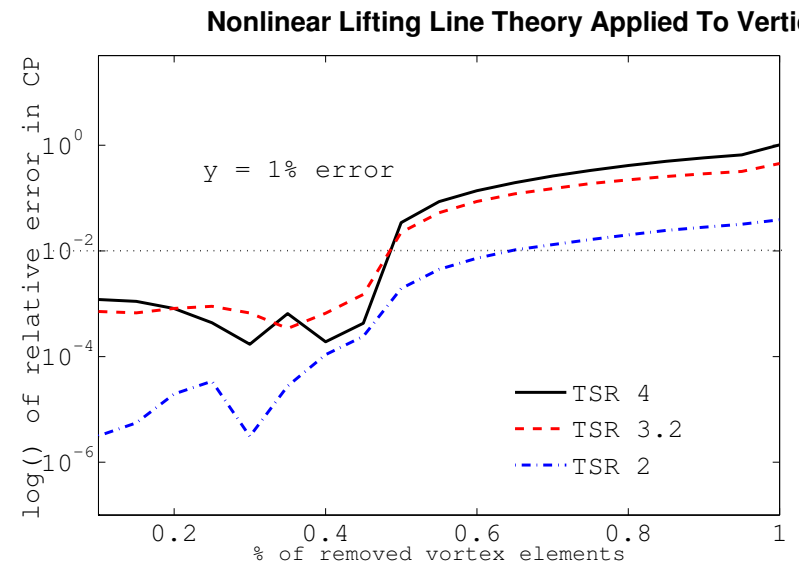

Figure 7. The effect of wake reduction on the simulation accuracy

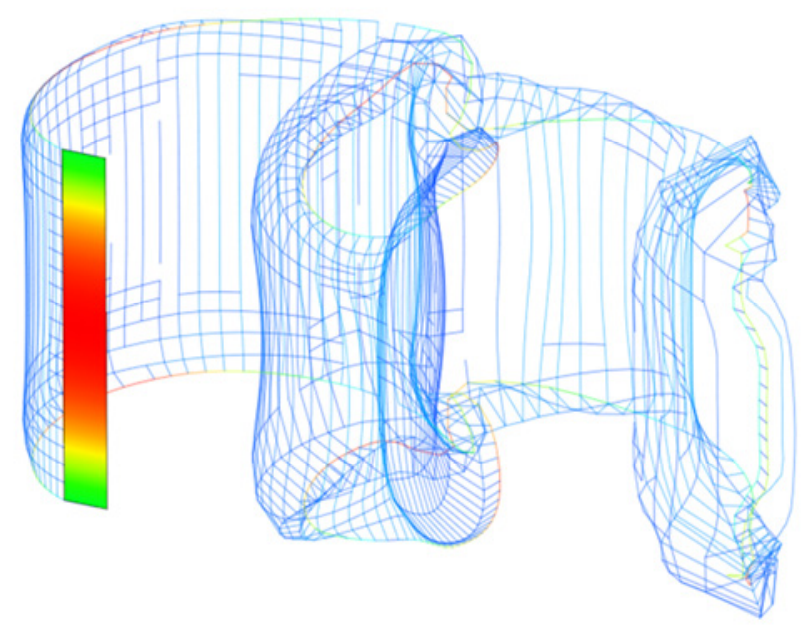

Figure 8. VAWT wake structure with $30 \%$ wake reduction

\section{VALIDATION / CODE COMPARISON}

This sections compares the quasi steady state performance predictions of the LLT method with those of a DMST model, already implemented in QBlade [11], and those of a 2D URANS simulation carried out at the University of Florence (described in [12; 13]). The rotor used in this comparison is a straight bladed $\mathrm{H}$-Darrieus rotor, simulated in a one bladed configuration (Table 1, Figure 8).

Table 1. H-Darieus reference rotor

\begin{tabular}{|ll|}
\hline Blade Number & 1 \\
Airfoil & NACA0018 \\
Radius & $0.85 \mathrm{~m}$ \\
Chord & 0.246 \\
Solidity & 0.144 \\
\hline
\end{tabular}

To compare the global results of the simulation methods graphs of the averaged power coefficient over the tip speed ratio are shown in Figure 10. Furthermore the local azimuthal distributions of the generated blade torque at three tip speed ratios, for a low, high and optimal induction case, are compared. The LLT simulation obtains the power curve via a ramp up simulation, varying the rotational speed at a constant $8 \mathrm{~m} / \mathrm{s}$ inflow. Adaptive time stepping is used to fix
Axis Wind Turbines: Development of a Practical Design Tool -5 the azimuthal discretization for all simulated cases to and angle of $5^{\circ}$.The main simulation parameters for the LLT are listed in Table 2.

Table 2. LLT Simulation parameters

\begin{tabular}{|ll|}
\hline Fixed Azimuthal & $5^{\circ}$ \\
discretization & 8 revolutions \\
Wake truncation & 0.3 \\
Wake reduction & 8 (sinusoidal spacing) \\
Number of blade elements & $8 \mathrm{~m} / \mathrm{s}$ \\
Inflow velocity & \\
\hline
\end{tabular}

The lift and drag polars used in the DMS and the LLT simulations are XFOIL [14] generated polars (NCRIT $=5$, forced transition at the leading edge) in the Reynolds number range between $5 \mathrm{e} 4$ and $1 \mathrm{e} 6$ (see Figure 9). Both codes interpolate within this Reynolds number range at every time step. The polars were extrapolated with the Viterna method, with a CD90 value of 1.85 . Dynamic stall effects are not modelled in the LLT \& DMS simulations.

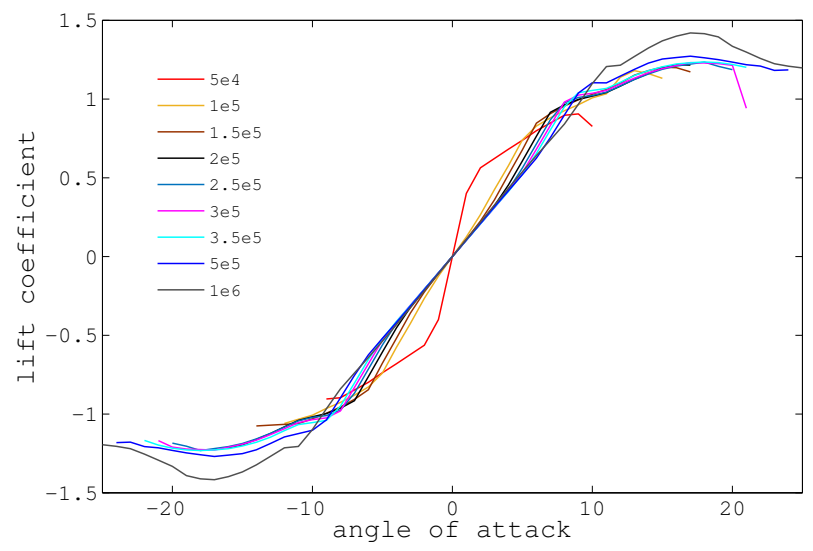

Figure 9. Lift polars used in the DMS \& LLT simulations

The CP over TSR curves for the 1 blade configuration in Figure 10 show considerably different agreement between the U-RANS, LLT and DMS predictions. The LLT results match the overall CP distribution best, while the DMS code with tip loss model under predicts, and without tip loss model over predicts, the rotor performance.

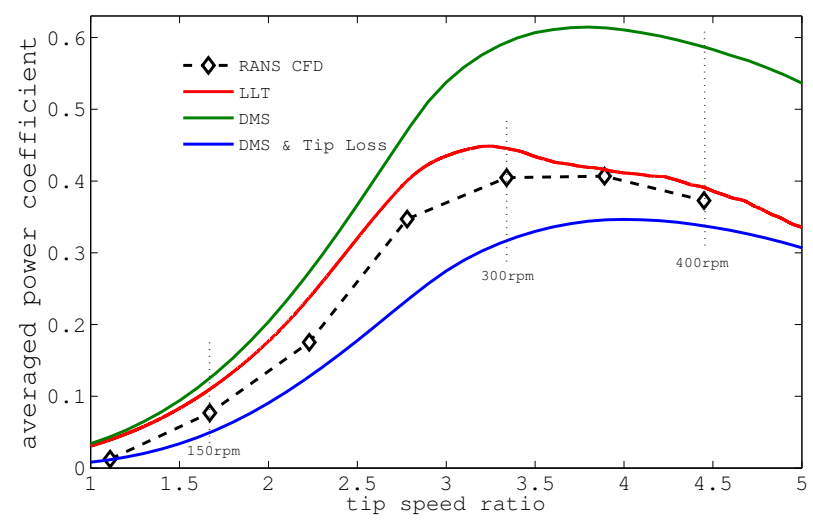

Figure 10. CP over TSR curves, 1 bladed configuration 
Figure 11 shows the azimuthal variation of the torque coefficient at a relatively low TSR of 1.67. The distributions differ greatly between URANS and the two engineering methods. Due to the low TSR of 1.67, the AoA varies between $+-35^{\circ}$ and the blade experiences deep stall during every rotation. Because no dynamic stall model is implemented in neither the LLT nor the DMS code these differences are to be expected. Again, it can be seen that the DMS tip loss model has a large effect on the predicted torque.

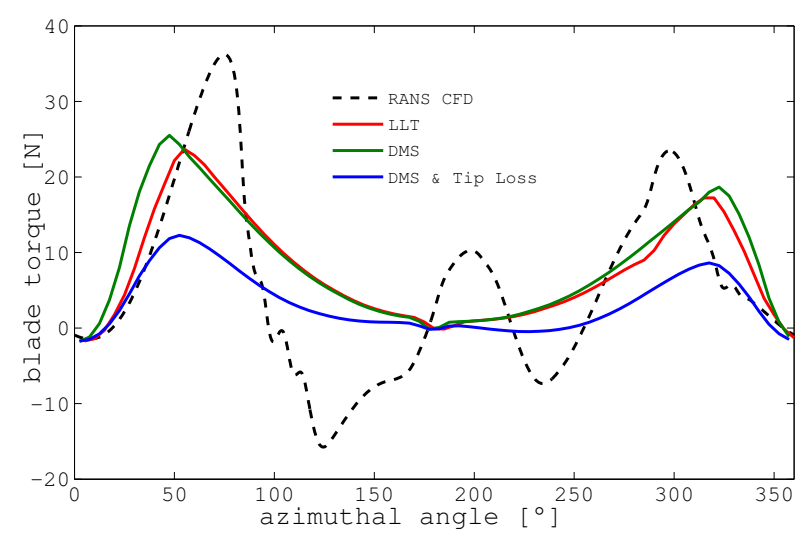

Figure 11. Torque coefficient over azimuthal angle @ 150rpm / TSR: 1.67, 1 bladed configuration

Bearing in mind that the set of polars used is plain XFoil data created without any special modifications or a dynamic stall model, the agreement for the torque coefficient in Figure 12 is surprisingly good between the URANS and the LLT code. The DMS code over- and under predicts the URANS results and also predicts the torque maxima at slightly lower azimuthal angles. The simulated TSR of 3.34 is near the maximum $\mathrm{CP}$ value, at this operational point the $\mathrm{AoA}$ varies between $+15^{\circ}$ and $-10^{\circ}$, thus dynamic stall effects are expected to play a less significant role.

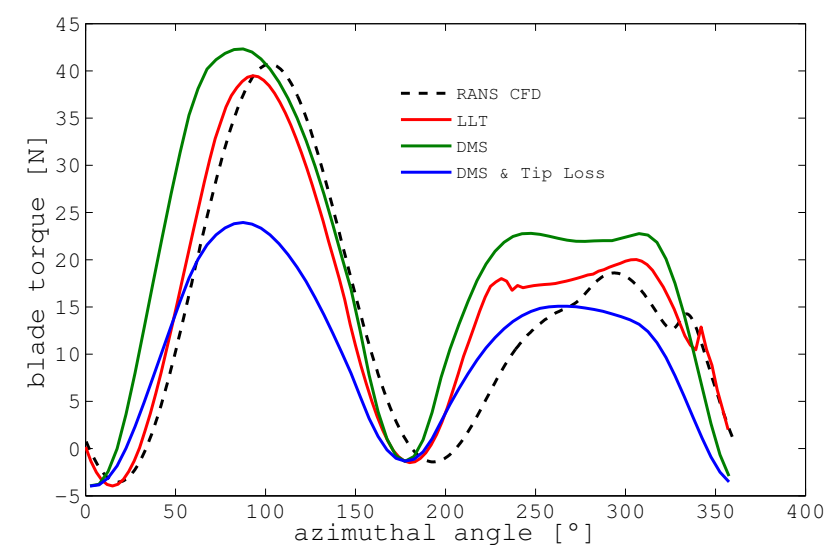

Figure 12. Torque coefficient over azimuthal angle @ 300rpm / TSR: 3.34, 1 bladed configuration

Figure 13 compares the torque profiles for a high induction case above the optimal TSR. The AoA at this operational point varies between $+8^{\circ}$ and $-7^{\circ}$ and dynamic stall effects play a minor role now. As a result the LLT prediction is slightly improved. The DMS results show the same trend for over and under prediction of torque dependent on the use of the tip loss model.

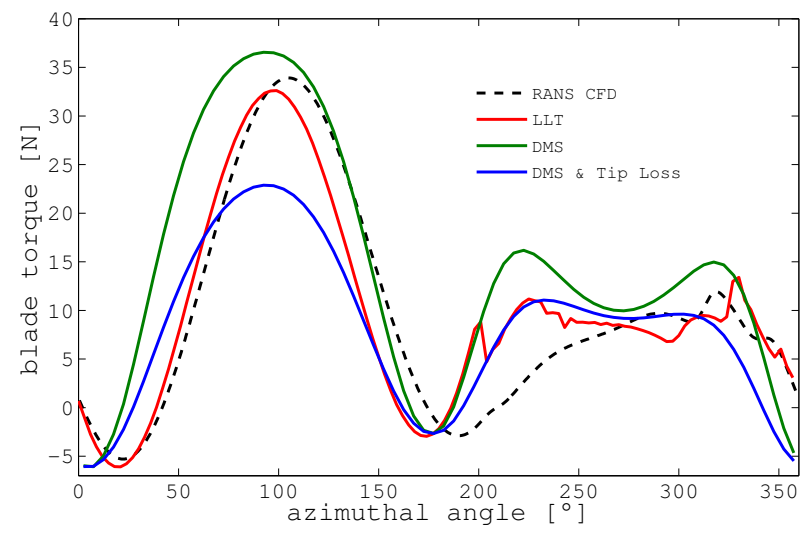

Figure 13. Torque coefficient over azimuthal angle @ 400rpm / TSR: 4.45, 1 bladed configuration

\subsubsection{Runtime Information}

As an estimate for the real life performance of the LLT: With the settings from Table 2, on an Intel $\AA^{\circledR}$ Xeon $\AA$ E3-1230V3, with $3.3 \mathrm{GHz}$ and 4 physical (+4 virtual) cores the simulation takes 137 seconds for 10 revolutions of the rotor (or 720 time steps). At these settings the wake is made up of roughly 7000 free wake elements. If, for the same settings the azimuthal discretization is increased to $10^{\circ}$, the simulation only takes 22 seconds (10 revolutions or 360 time steps) with roughly 3500 free wake elements present in the domain.

Recently, after these simulations have been performed, the OpenCL [15] framework was implemented to evaluate the Biot-Savart equation in parallel on GPUs. This massive parallelization decreases the needed computing time by a factor of up to 30 , as demonstrated in [16].

\section{CONCLUSION AND OUTLOOK}

A Lifting Line Free Vortex Wake code, optimized for VAWT simulations, was implemented in the wind turbine design suite QBlade. During the implementation special emphasis was placed on achieving a high robustness and computational efficiency of the code and some of the measures taken have been discussed in the previous sections. A validation and comparison to a URANS and DMS simulations shows that the LLT code generates reasonable results for "steady state" performance simulations. However it was also found that the code is lacking a dynamic stall model (by now a BeddoesLeishman type dynamic stall model has been implemented and validated, see $[17 ; 18])$. Furthermore the impact of polar data quality and different polar extrapolation techniques on the simulation results needs to be further investigated to increase the confidence of simulation results. However the present LLT model is a promising starting point towards a fully coupled aero-elastic simulation environment for VAWT.

\section{REFERENCES}

[1] H. J. Sutherland, D. E. Berg and T. D. Ashwill. A Retrospective of VAWT Technology. Sandia Report, SAND2012-0304, 2012 
[2] I. Paraschivoiu, Wind Turbine Design - With Emphasis on Darrieus Concept, Presses Internationales Polytechnique

[3] S. C. Mclntosh, H. Babinsky and T. Bertényi, Convergence Failure and Stall Hysteresis in ActuatorDisk Momentum Models Applied to Vertical Axis Wind Turbines. Journal of Solar Energy Engineering 131, 034502, 2009

[4] C. J. Simão Ferreira, The near wake of the VAWT, 2D and $3 \mathrm{D}$ views of the VAWT aerodynamics, $\mathrm{PhD}$ Thesis, Delft University of Technology, Delft, The Netherlands, 2009.

[5] D. Marten, M. Lennie, G. Pechlivanoglou, C. N. Nayeri and C. O. Paschereit, Implementation, Optimization and Validation of a Nonlinear Lifting Line Free Vortex Wake Module Within the Wind Turbine Simulation Code QBlade, Proceedings of the ASME Turbo Expo 2015, 2015

[6] D. Marten, QBlade Guidelines v0.9, Technical Report, TU Berlin, 2015

[7] A. Van Garrel, Development of a wind turbine aerodynamics simulation module, Technical Report, ECN, 2003

[8] M. H. M. Kloosterman, Development of the Near Wake behind a Horizontal Axis Wind Turbine, Masters Thesis, TU Delft, 2009

[9] $\mathrm{H}$. Abedi, Development of Vortex Filament Method for Aerodynamic Loads on Rotor Blades, Masters Thesis, Chalmers University, Sweden, 2013.

[10] B. Montgomerie, Vortex Model for Wind Turbine Loads and Performance Evaluation, Scientific Report, FOI-R-1301-SE, Swedish Defence Research Agency, 2004

[11] D. Marten, M. Lennie, G. Pechlivanoglou, C. N. Nayeri and C. O. Paschereit, Development and application of a simulation tool for vertical and horizontal axis wind turbines, Proceedings of ASME Turbo Expo 2013, San Antonio, Texas, USA
[12] F. Balduzzi, A. Bianchini, R. Maleci, G. Ferrara and L. Ferrari, Blade design criteria to compensate the flow curvature effects in $\mathrm{H}$-Darrieus wind turbines, Journal of Turbomachinery, 2014

[13] F. Balduzzi, A. Bianchini, R. Maleci, G. Ferrara and L. Ferrari, Critical issues in the CFD simulation of Darrieus wind turbines, Renewable Energy, 85, 2016

[14] M. Drela, M. Giles, Viscous-Inviscid Analysis of Transonic and Low Reynolds Number Airfoils, AIAA Journal Vol.25, No.10, 1989

[15] J. E. Stone, D. Gohara, G. Shi, OpenCL: A Parallel Programming Standard for Heterogeneous Computing Systems, Computing in Science \& Engineering, Volume 12, Issue 3, 2010

[16] D. Marten, G. Pechlivanoglou, C.N. Nayeri, C.O. Paschereit, Integration of an Unsteady Nonlinear Lifting Line Free Vortex Wake Algorithm in a Wind Turbine Design Framework, in Proceedings of: EWEA Annual Meeting 2015, Paris, France

[17] D. Marten, A. Bianchini, et al., Effects of Airfoils' Polar Data in the Stall Region on the Estimation of Darrieus Wind Turbine Performance, GT2016-56685, Accepted for: ASME Turbo Expo 2016, Seoul, South Korea

[18] J. Wendler, D. Marten, G. Pechlivanoglou, C.N. Nayeri, C.O. Paschereit, Implementation and Validation of an Unsteady Aerodynamics Model for Horizontal and Vertical Axis Wind Turbines Within the Simulation Tool QBlade, GT2016-57184, Accepted for: ASME Turbo Expo 2016, Seoul, South Korea 